\title{
Iris Recognition Using Fuzzy Level Set and GEFE
}

\author{
Brian O'Connor, Kaushik Roy, Joseph Shelton, and Gerry Dozier
}

\begin{abstract}
This paper presents an efficient algorithm for iris recognition using the spatial fuzzy clustering with level set method, and genetic and evolutionary feature extraction techniques. The novelty of this research effort is that we deploy a fuzzy c-means clustering with level set (FCMLS) method in an effort to localize the nonideal iris images accurately. The FCMLS method incorporates the spatial information into the level set-based curve evolution approach and regularizes the level set propagation locally. The proposed iris localization scheme based on FCMLS avoids the over-segmentation and performs well against blurred iris/sclera boundary. Furthermore, we apply a genetic and evolutionary feature extraction (GEFE) technique, which uses genetic and evolutionary computation to evolve modified local binary pattern (MLBP) feature extractor to elicit the distinctive features from the unwrapped iris images. The MLBP algorithm combines the sign and magnitude features for the improvement of iris texture classification performance. The identification and verification performance of the proposed scheme is validated using the CASIA version 3 interval dataset.
\end{abstract}

Index Terms - Iris recognition, fuzzy c-means clustering, level set, modified local binary pattern, and genetic and evolutionary feature extraction.

\section{INTRODUCTION}

Automated person identification systems based on iris biometrics have gained immense popularity due to its applicability to many areas, including national border control, forensics and secure financial transactions. A large number of iris recognition algorithms mainly depend on the ideal iris images that are captured in an ideal situation to ensure the higher performance [1]-[6]. However, in many cases, iris image acquisition process may be affected by different nonideal factors such as illumination variations, noncooperation of persons, head rotations, gaze directions, and camera angles. These undesirable factors in data acquisition process may result in motion blurs, reflections, eyelash and eyelid occlusions, and pupil center deviation and further hamper the segmentation performance. Iris recognition using such noisy iris images still remains a challenging issue.

Several attempts have been made for nonideal iris recognition based on active contours. In [6], inner and outer boundaries were isolated using the discrete Fourier series

Manuscript received October 30, 2013; revised January 4, 2014. This research was supported in part by the Army Research Laboratory (ARL) for the multi-university, Center for Advanced Studies in Identity Sciences (CASIS) and by the National Science Foundation (NSF), Science \& Technology Center: Bio/Computational Evolution in Action Consortium (BEACON).

Brian O'Connor, Kaushik Roy, Joseph Shelton, and Gerry Dozier are with the Computer Science Department, North Carolina Agricultural and Technical State University, Greensboro, NC 27411 USA (e-mail: bpoconno@aggies.ncat.edu; kroy@ncat.edu; jashelt1@aggies.ncat.edu; gvdozier@ncat.edu). expansions of the contour data. The modified Mumford-Shah segmentation model was deployed in [2] to localize the iris and pupil boundary. A traditional active contour model and the Hough transform technique were applied to segment the iris region in [7]. The Geodesic Active Contours (GAC) were employed in [8] to isolate iris information from the off-angle iris images. The parametric active contour-based curve evolution process may be interrupted at a certain local minima such as the specular reflections, the thick radial fibres in the iris or the crypts in the ciliary region [8]. The active contours with an edge stopping function as halting criteria may fail to isolate the weak outer boundary [2], [8]. Addressing the above problems, we propose to apply the Fuzzy C-Means clustering with Level Set (FCMLS) method which utilizes fuzzy clustering and the Level Set (LS) method for optimal estimation of the iris contour [9]. The fuzzy clustering utilizes the spatial information along with edge stopping criteria during a flexible curve propagation process. The controlling parameters of LS segmentation are calculated from the fuzzy clustering during the curve evolution process. Using fuzzy clustering, the LS method can also be regularized. This discerns FCMLS from the other active contour-based iris localization methods [9]. The LS and fuzzy clustering methods have been used extensively on medical images. However, in this effort, we demonstrate the application of the fuzzy LS algorithm in the area of iris segmentation.

Feature extraction is a key issue in iris recognition. Different researchers exploited several iris feature extraction techniques, including Gabor wavelets, Daubechies wavelets, and discrete cosine transform [1]-[7]. Local Binary Pattern (LBP) has been regarded as one of the most robust local texture descriptor, and it has been shown promising performance in the computer vision field, including motion analysis and face recognition [10]. In this work, we apply a modified version of LBP, denoted hereafter MLBP, to represent the iris texture [11]. In MLBP, a local region is represented by its center pixel and a local difference of Sign-Magnitude Transform (SMT). The local difference of SMT is then decomposed into two complementary components: difference sign and difference magnitude [11]. We also apply a hybrid feature extraction/genetic and evolutionary technique known as Genetic and Evolutionary Feature Extraction (GEFE). This technique uses Genetic and Evolutionary Computations (GECs) [12]-[15] to evolve the dimensions and locations of regions in MLBP. The fitness function of GEFE is focused on identification accuracy so regions are evolved to extract from the most discriminating areas of an image while reducing the number of regions needed to be extracted from. GEFE has previously been applied towards facial recognition [12], [13] as well as fly wing gender classification [15] and has outperformed the traditional feature extractor. In this paper, we fuse both the 
sign and magnitude features in order to obtain a better iris texture classification performance. We then apply GEFE with MLBP toward iris feature extraction to further improve the performance.

The rest of this paper is organized as follows. Section II describes iris segmentation approach. The MLBP and GEFE are discussed in Section III. Section IV reports the experimental results and Section V provides our conclusions.

\section{SEGMENTATION OF IRIS IMAGES}

Iris localization of the noisy iris image is a complex task since the shapes of the iris and pupil are not exactly circular or elliptical. The iris contour may differ depending on the image acquisition techniques [2]-[5]. We divide the iris segmentation process into three steps. In the first step, we apply a reflection detection process. We use simple image processing techniques and elliptical model to approximate the inner (pupil) boundary of the iris in the second step. In the final step, we apply the FCMLS to find the exact outer boundary of the iris based on the estimated boundary obtained in the previous step.

The strong reflection area is identified, in the first stage of segmentation, based on the method reported in [16]. A simple thresholding approach is applied to find the strong reflection area. A pixel with an intensity value higher than a certain threshold belongs to the strong reflection area. The reflection is found by the following inequality [16]:

$$
g(x, y)>T_{s}
$$

where $g(x, y)$ denotes the intensity of an image at the point $(x, y)$ and $T_{S}$ is the required threshold value. A weak reflection area denotes the transition from a strong reflection to the iris region. We apply the following statistical inequality to detect the weak reflection points [16]:

$$
\mu+\gamma \sigma<g(x, y)
$$

where $\mu$ and $\sigma$ are the mean and standard deviation for the distribution of the intensity in the iris image $g(x, y)$, respectively, and $\gamma$ denotes the control parameter. Any pixel that is close to the strong reflection area and that satisfies (2) is considered as a weak reflection. Fig. 1(b) exhibits the reflection detection result. The pupil is usually the darkest object in an image of an eye, which allows for easier detection. In the second step, we use a simple binary thresholding to find the pupil area as shown in Fig.1(c). After applying the morphological closing operations and determining the largest connected region to obtain the pupil area, most of the unwanted regions are removed (see Fig.1(d)). Prior to applying the curve evolution approach based on FCMLS, we deploy Direct Least Square (DLS) elliptical fitting technique to approximate the pupil boundary [3], [4]. To find an approximation of the inner boundary, an elliptical region with the five parameters $\left(p_{1}, p_{2}\right.$, $\left.r_{1}, r_{2}, \varphi_{1}\right)$ is selected, and the intensity values are measured for a fixed number of points on the pupil circumference. We vary the ellipse parameters with a small step size of three pixels to increase the ellipse size, and choose a fixed number of points randomly on the circumference to calculate the total intensity value. We repeat this process to find the boundary with a maximum variation in luminance and the center of the pupil. Fig. 1(e) shows the approximated pupil boundary. Based on the approximation of the inner boundary, the curve is evolved by using the fuzzy level set for accurate segmentation of the iris region [9]. In the following paragraphs, we briefly discuss the segmentation process based on fuzzy level set method.

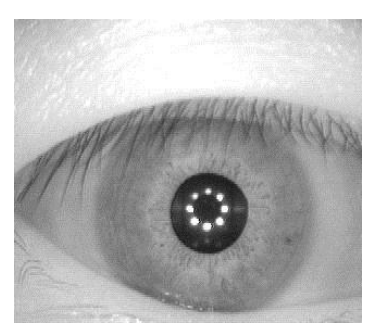

(a)

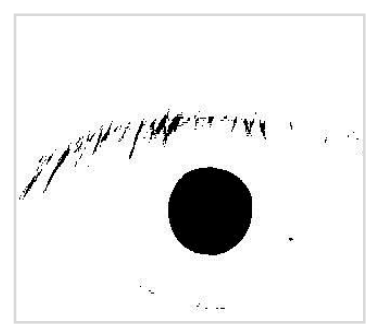

(c)

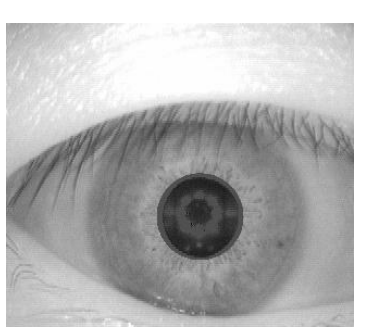

(b)

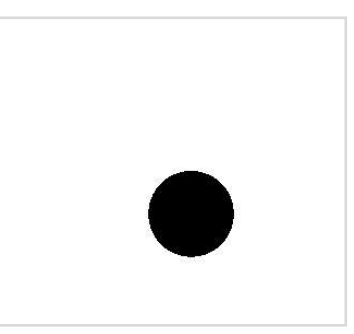

(d)

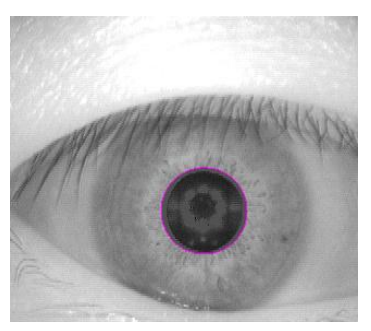

(e)

Fig. 1. (a) The original image from the CASIA version 3 interval dataset, (b) reflection elimination, (c) image after binary thresholding, (d) application of morphological closing operation, (e) approximation of pupil boundary using DLS elliptical fitting.

Let us briefly discuss the fuzzy clustering technique first. The geometric center and the scope of each subclass are calculated adaptively to reduce the cost function in fuzzy c-means clustering [9]. This method employs the membership function $\mu_{m n}$ to find the degree of membership of $n$th object to the $m$-th cluster. The cost function in fuzzy c-means clustering is [9]

$$
J=\sum_{n=1}^{N} \sum_{m=1}^{C} \mu_{\mathrm{mn}}^{\mathrm{i}}\left\|i_{n}-v_{m}\right\|^{2}
$$

where, $i_{n}$ is the image pixel, $v_{m}$ is the center value of $m$-th cluster, $N$ presents the total number of image pixels and $C$ denotes total clusters. $l(>1)$ controls the fuzziness of the resultant segmentation. The membership functions are accountable to the following constraints:

$$
\sum_{m=1}^{C} \mu_{m n}=1 ; 0 \leq \mu_{m n} \leq 1 ; \sum_{n=1}^{N} \mu_{m n}>0
$$

The membership functions and the geometric centers can be updated in each iteration as follows: 


$$
\begin{gathered}
\mu_{m n}=\frac{\left\|i_{n}-v_{m}\right\|^{-2 /(l-1)}}{\sum_{k=1}^{C}\left\|i_{n}-v_{k}\right\|^{-2 /(l-1)}} \\
v_{i}=\frac{\sum_{n=1}^{N} \mu_{m n}^{l} i_{n}}{\sum_{n=1}^{N} \mu_{m n}^{l}}
\end{gathered}
$$

In order to incorporate the spatial information into the fuzzy clustering technique, Chuang et al. [17] proposed the following fuzzy membership functions

$$
\mu_{m n}^{\prime}=\frac{\mu_{m n}^{p} h_{m n}^{p}}{\sum_{k=1}^{C} \mu_{k n}^{p} h_{k n}^{p}}
$$

where, $p$ and $q$ control the respective contributions and the variable $h_{m n}$ includes the spatial information [9], [17]. To improve the iris segmentation performance in a noisy situation, the spatial fuzzy clustering approach is applied with the LS method. Unlike the fuzzy clustering method, in which the pixel classification is used, LS methods deploy dynamic variational boundaries for image segmentation [9]. The spatial fuzzy clustering technique is utilized to initiate the LS segmentation, estimate controlling parameters and regularize level set evolution. The fuzzy LS algorithm, proposed in [9], deploys the automatic initialization and parameter configuration of the LS localization, using spatial fuzzy clustering results. In the LS formulation, the active contours, denoted by $C$, can be represented by the zero $\operatorname{LS} C(t)=$ $\{(x, y) \mid \phi(t, x, y)=0\}$ of a LS function $\phi(t, x, y)$. To evolve the curve towards the outer boundaries, we use the following total energy functional according to [18]:

$$
\varepsilon(\phi)=\mu \rho(\phi)+\varepsilon_{g, \lambda, v}(\phi)
$$

where $\varepsilon_{g, \lambda, v}(\phi)$ denotes the external energy, which depends on the image data and drives the zero LS towards the iris boundaries, and $\mu \rho(\phi)(\mu>0)$ denotes the internal energy, which penalizes the deviation of $\phi$ from the Signed Distance Function (SDF) during the evolution and is defined as [18]:

$$
\rho(\phi)=\int_{\Omega} \frac{1}{2}(|\nabla \phi|-1)^{2} d x d y
$$

where $\Omega$ is the image domain. In (8), $g$ denotes the edge detector function and is defined by:

$$
g=\frac{1}{1+\left|\nabla G_{\sigma} * I\right|^{2}}
$$

where $G_{\sigma}$ is the Gaussian kernel with a standard deviation denoted as $\sigma$, and $I$ denotes an iris image. We can further define the external energy term $\varepsilon_{g, \lambda, v}(\phi)$ of (8) as:

$$
\varepsilon_{g, \lambda, v}(\phi)=\lambda L_{g}(\phi)+v A_{g}(\phi)
$$

where $\lambda>0$ and $v$ are constants, and the terms $L_{g}(\phi)$ and $A_{g}(\phi)$ in (11) are respectively defined by [18]:

$$
L_{g}(\phi)=\int_{\Omega} g \delta(\phi)|\nabla \phi| d x d y
$$

and

$$
A_{g}(\phi)=\int_{\Omega} g H(-\phi) d x d y
$$

where $\delta$ is the univariate Dirac function, and $H$ is the Heaviside function. The energy functional $L_{g}(\phi)$ measures the length of the zero LS curve of $\phi$, and $A_{g}(\phi)$ is used to speed up the curve evolution. From the calculus of variations, the Gateaux derivative of the functional $\varepsilon$ in (11) can be written as:

$$
\begin{gathered}
\frac{\partial \varepsilon}{\partial \phi}=-\mu\left[\Delta \phi-\operatorname{div}\left(\frac{\nabla \phi}{|\nabla \phi|}\right)\right]-\lambda \delta(\phi) \operatorname{div}\left(g \frac{\nabla \phi}{|\nabla \phi|}\right) \\
-\operatorname{vg} \delta(\phi)
\end{gathered}
$$

where $\Delta$ is the Laplacian operator. The function $\phi$ that minimizes this functional satisfies the Euler-Lagrange equation $\frac{\partial \varepsilon}{\partial \phi}=0$. Next, the evolution equation of the LS function, where the spatial information can be incorporated, is defined as [18]:

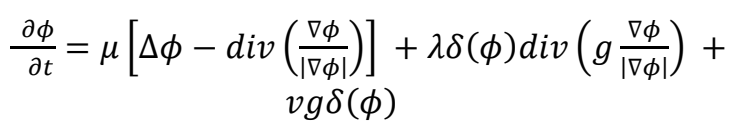

The second and third terms on the right-hand side of (15) represent the gradient flows of the energy functional and are responsible for driving the zero level-curve towards the outer boundary of the iris. The Dirac function $\delta(x)$ in $(15)$ is defined by:

$$
\delta_{\varepsilon}(x)= \begin{cases}0, & |x|>\varepsilon \\ \frac{1}{2 \varepsilon}\left[1+\cos \left(\frac{\pi x}{\varepsilon}\right)\right], & |x| \leq \varepsilon\end{cases}
$$

The LS algorithm eliminates the computationally expensive re-initialization for SDFs and the evolution process may start from an arbitrary binary region:

$$
\phi_{0}(x, y)=\left\{\begin{array}{c}
-C, \phi_{0}(x, y)<0 \\
C, \text { otherwise }
\end{array}\right.
$$

The applied FCMLS engages fuzzy clustering with spatial restrictions to detect the iris contours in an image. Profiting from the flexibility in (17), the fuzzy clustering results can certainly improve the LS evolution process. If the intriguing factor in fuzzy clustering results produce $\boldsymbol{R}_{k}:\left\{r_{k}=\mu_{n k}, n=\right.$ $\left.x \times N_{y}+y\right\}$ [9], we can find it advantageous to start the LS function as

$$
\phi_{0}(x, y)=-4 \varepsilon\left(0.5-\mathbf{B}_{\mathrm{k}}\right)
$$

where $\varepsilon$ is a constant and used to adjust the Dirac function as defined in (16). $\boldsymbol{B}_{k}$ is a binary image retrieved from

$$
\mathbf{B}_{k}=\mathbf{R}_{k} \geq b_{0}
$$

where $b_{0}(\in(0,1))$ is an adjustable threshold. FCMLS method takes the degree of membership for each pixel $\mu_{k}$ as the distance to component of interest $\boldsymbol{R}_{\boldsymbol{k}}$. In order to push or pull the dynamic interface towards the iris region, a balloon force is applied as follows: 


$$
G\left(\mathbf{R}_{k}\right)=1-2 \mathbf{R}_{k}
$$

The resulting matrix has a variable pulling or pushing force at each image pixel. The LS curve now can evolve towards the iris boundary regardless its initial position. The required evolutionary function of (15) can be transformed into

$$
\begin{gathered}
\frac{\partial \phi}{\partial t}=\mu\left[\Delta \phi-\operatorname{div}\left(\frac{\nabla \phi}{|\nabla \phi|}\right)\right]+\lambda \delta(\phi) \operatorname{div}\left(g \frac{\nabla \phi}{|\nabla \phi|}\right) \\
+g G\left(\mathbf{R}_{k}\right) \delta(\phi)
\end{gathered}
$$

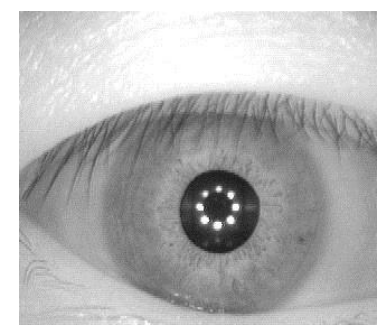

(a)

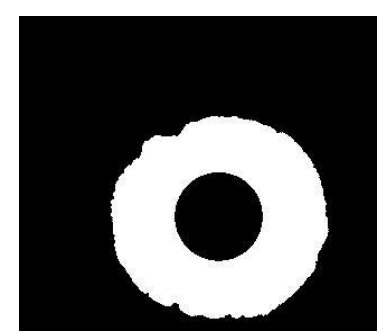

(c)

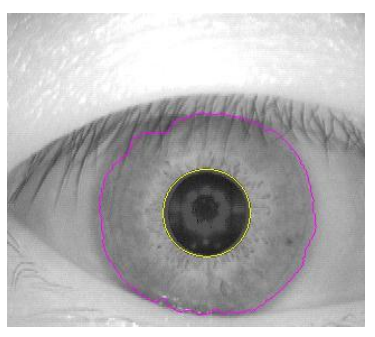

(b)

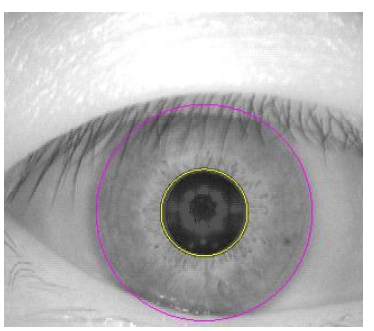

(d)

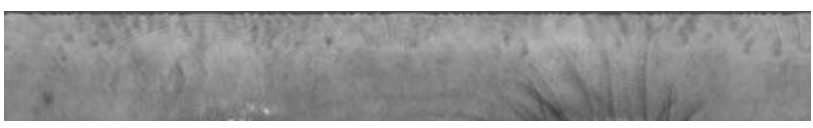

(e)

Fig. 2. (a) Original image from the CASIA version 3 interval dataset, (b) pupil and iris detection using the FCMLS model, (c) binary masking of the iris with FCMLS, (d) iris image after applying the circle fitting technique, and (e) normalized image.

Eq. (21) can be used to evolve the curve in an effort to detect the iris boundary. Using spatial fuzzy clustering, the balloon force can be calculated. Also, the LS evolution can be adapted to the distance of the iris contour. Fig. 2(b) shows the FCMLS-based curve evolution process. Since the elicited iris regions are not exactly circular and elliptical and may be of arbitrary shapes (See Fig. 2(c)), a circle fitting strategy is applied to the extracted non-circular iris region in an attempt to mitigate the size irregularities as shown in Fig. 2(d) [3]. To convert iris region to a rectangular form, the centre values obtained through the circle fitting process and the approximated radius of such a circle are used for the unwrapping process. We unwrap the iris region to a normalized rectangular block with a fixed dimension of size $64 \times 512$ using the rubber-sheet model [6]. Fig. 2(e) shows the unwrapped image.

\section{GENETIC AND EVOLUTIONARY FEATURE EXTRACTION WITH MODIFIED LOCAL BINARY PATTERN}

Texture classification plays an important role in the area of iris recognition. The LBP operator, proposed by Ojala $e t$ al.
[10], is an effective way of texture description. LBP has been applied to many other applications, including face recognition, dynamic texture recognition and shape localization [11]. In this work, we apply MLBP operator with the GEFE to extract the iris features.

\section{A. Modified Local Binary Pattern}

In the traditional LBP operator, a pixel of an image is compared with its neighboring pixels [10]:

$$
L B P_{P, R}=\sum_{p=0}^{p-1} r\left(g_{p}-g_{c}\right) 2^{p}, r(x)=\left\{\begin{array}{l}
1, x \geq 0 \\
0, x<0
\end{array}\right.
$$

where $g_{c}$ indicates the gray level value of the center pixel, $g_{p}$ represents the value of the neighboring pixels of the center, $P$ denotes the total number of neighboring pixels and $R$ is the radius of the neighborhood. For an image of size $I^{*} J$, the LBP pattern is measured for each pixel of an image and a histogram is created to represent the iris texture [11]:

$$
\begin{gathered}
\operatorname{Hist}(k)=\sum_{i=1}^{I} \sum_{j=1}^{J} f\left(L B P_{P, R}(i, j), k\right), k \in[0, K] \\
f(x, y)=\left\{\begin{array}{c}
1, x=y \\
0, \text { otherwise }
\end{array}\right.
\end{gathered}
$$

where $K$ is the the maximal LBP pattern value. We compute the number of spatial changes (bitwise $0 / 1$ changes) in the LBP as follows:

$$
\begin{aligned}
\operatorname{Unified}\left(\operatorname{LBP}_{P, R}\right) & \begin{aligned}
& \\
&=\left|r\left(g_{p-1}-g_{c}\right)-r\left(g_{0}-g_{c}\right)\right| \\
&+\sum_{p=1}^{p-1}\left|r\left(g_{p}-g_{c}\right)-r\left(g_{p-1}-g_{c}\right)\right|
\end{aligned}
\end{aligned}
$$

In the unified LBP pattern, limited transition (Unified $\leq$ 2 ) is presented in the circular binary LBP code pattern and measured as:

$$
\begin{gathered}
L B P_{P, R}^{\text {Unified }}=\sum_{p=0}^{p-1} r\left(g_{p}-g_{c}\right), \\
\text { if Unified }\left(L B P_{P, R}\right) \leq 2
\end{gathered}
$$

Now, the local difference, Dist $_{P}$ is calculated between the center pixel $g_{c}$ and the evenly spaced neighboring pixels, $g_{p}, p=0,1,2, \ldots, P-1$ as $\operatorname{Dist}_{P}=g_{p}-g_{c}$. Thus, the local image structure at $g_{c}$ with the local difference vector $\left[\right.$ Dist $_{0}, \ldots \ldots$, Dist $\left._{p-1}\right]$ is created. The local difference vector provides robust performance against the illumination changes as the center intensity value, $g_{c}$ is removed. We decompose the Dist $_{P}$ into two components [11]:

$$
\operatorname{Dist}_{P}=s_{p} * m_{p} \text { and }\left\{\begin{array}{c}
s_{p}=\operatorname{sign}\left(\text { Dist }_{P}\right) \\
m_{p}=\mid \text { Dist }_{P} \mid
\end{array}\right.
$$

where $s_{p}=\left\{\begin{array}{c}1, \text { Dist }_{P} \geq 0 \\ -1, \text { Dist }_{P}<0\end{array}\right.$ and $m_{P}$ are the sign and 
magnitude of Dist $_{P}$, respectively. In this research effort, we utilize the both the sign and magnitude components of Dist $_{P}$ in an effort to improve the iris feature extraction performance. The (25) denotes the local difference, SMT. To obtain the iris texture features, first, we divide the normalized iris region into several patches and apply the MLBP on each patch (see Fig. 3). Each patch is represented by 256 sign and 256 magnitude components since we consider all LBP patterns in this effort. We concatenate the sign and magnitude components and thus, present a single patch by $256 \times 2=512$ components. In the next section, we discuss the genetic and evolutionary feature extraction method which is also applied with the MLBP.

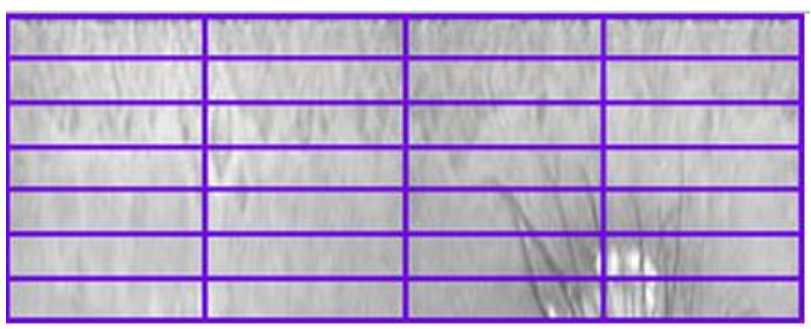

Fig. 3. Normalized image is divided into 28 sub-images.

\section{B. Genetic and Evolutionary Feature Extraction}

Genetic and Evolutionary Feature Extraction (GEFE) is a technique that evolves MLBP-based Feature extractors (FEs) [12]-[15]. Unlike a MLBP FE, GEFE evolves FEs that can have patches of varying sizes in various positions on an image. Because GEFE is an instance of a Genetic and Evolutionary Computation, a FE must be represented as a candidate solution. We use a 6-tuple with 5 sets and 1 single value, represented as $\left(<X_{i}, Y_{i}, W_{i}, H_{i}, M_{i}, f_{i}>\right)$. Each of the patches in a particular $\mathrm{FE}, f e_{i}$, are designed using the values in the 6-tuple. The $X_{i}$ and $Y_{i}$ sets hold the $\langle X, Y\rangle$ points of the center of each patch in $f e_{i}$, while the sets $W_{i}$ and $H_{i}$ hold the width and heights of the matches. The set $M_{i}$ denotes a masking value for each patch in $f e_{i}$. Though there can be multiple patches defined by the 6-tuple, a patch's specific masking value determines whether the features extracted by that patch are included in the resulting FV.

The fitness, $f_{i}$, is determined by applying $f e_{i}$ towards a dataset of subject's iris images. A subject has a number of images that vary, and these images are separated into a probe set and a gallery set. The $f e_{i}$ is applied on these images to create FVs, and the FVs in the probe set are compared to all of the FVs in the gallery set using the Manhattan distance measure [13]. The two FVs that have the smallest Manhattan distance are considered to be matched. If a probe FV is incorrectly matched with a gallery $\mathrm{FV}$, then $f e_{i}$ is said to cause an error. The $f e_{i}$ also considers how much surface area of the image it covers. The resulting $f_{i}$ is the number of errors $e$ added to the percent of patches activated $a$, shown below in (27).

$$
f_{i}=10 e+a
$$

\section{EXPERIMENTAL RESULTS AND DISCUSSION}

We conducted the experiments on the CASIA version 3 interval dataset [19]. The CASIA version 3 interval iris dataset includes 2639 iris images from 249 different persons, with 396 iris classes. Most of the images were captured in two sessions with at least one month interval. The iris images are 8-bit gray level images with a resolution of $320 \times 280$. Since the main focus of this paper is to propose a new segmentation technique along with a feature extraction method, we used our method to segment the entire dataset. We applied the FCMLS to localize the iris images. We conducted an extensive set of experiments and selected the control parameter values of FCMLS as follows: template radius $=5$, spatial filter weight, $\sigma=2$, fuzzy thresholding $=$ 0.25 , Dirac regulator, $\varepsilon=1.5$, and number of iterations, $T=150$. These parameter values allow us to control the growth of the LS contour as well as the clustering classifications. Since we used flexible contour-based approach for iris detection, we did not include eyelid detection method. Fig. 4 shows the segmentation results and Fig. 5 shows some normalized images. We find from Fig. 4 that our segmentation scheme performs well for the cases where the iris and sclera regions are separated by a weak boundary. Our proposed segmentation scheme exhibits an encouraging performance in nonideal situations. The moving front of boundary points of the evolving active contour may stop if a sudden variation in the intensity level occurs in the iris region due to a noisy pixel data. However, in our proposed method, the other boundary points continue to move and, hence, the curve evolution process, using FCMLS, keeps propagating towards the outer boundaries.
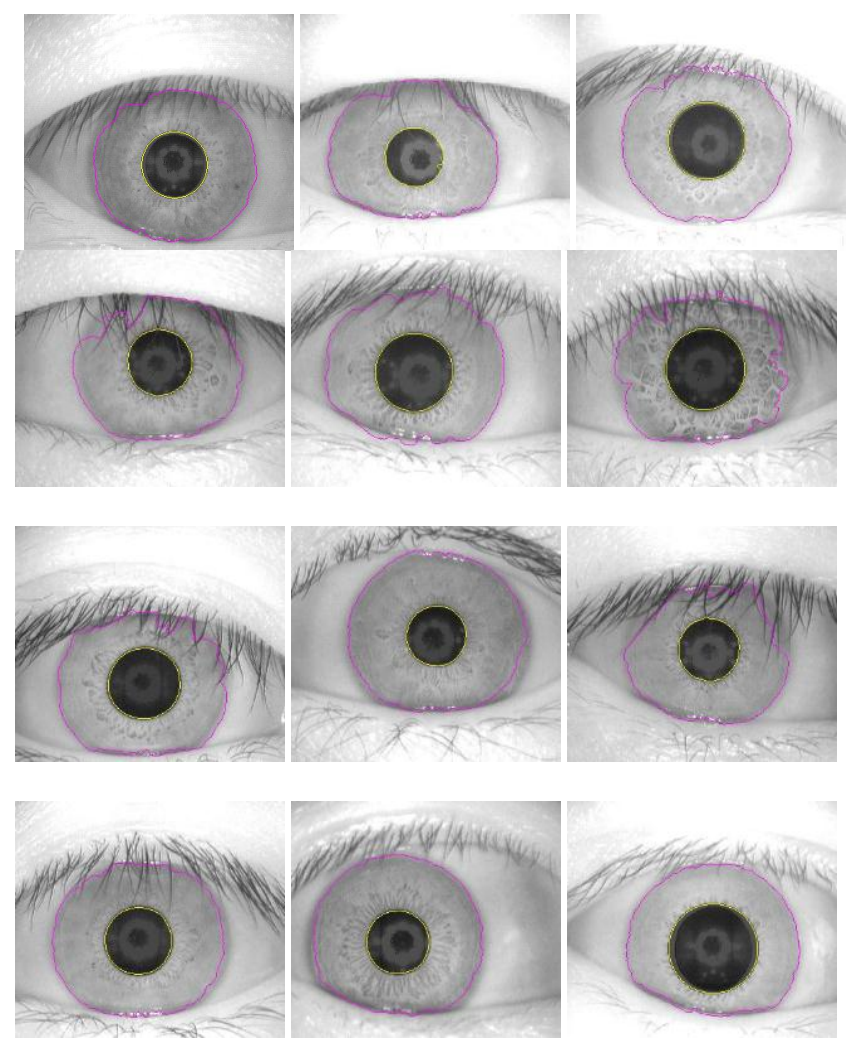

Fig. 4. FCMLS-based segmentation results on CASIA version 3 interval dataset.

GEFE with MLBP was run on the CASIA version 3 interval iris dataset 30 times for 1000 function evaluations using an Estimation of Distribution Algorithm (EDA). The population size was 20 and we used an elitist value of 1 . We ran an exhaustive search of the best partitioning of patches on 
the dataset and found that 6 patch rows by 13 patch columns was the best partition, for a total of 7 patches (See Fig. 6(a)). The recognition results of MLBP and its GEFE variant (GEFE_MLBP) are shown in Table I, as well as the number of patches used by each method. The second and third columns denote the best performance and number of patches used by MLBP and GEFE_MLBP. The average recognition accuracy and average number of patches used by GEFE_MLBP are shown in the third and fourth columns respectively. The best accuracy of MLBP was $86 \%$ whereas the average recognition accuracy for GEFE-MLBP was $88.42 \%$ and the best accuracy of all 30 genetically evolved feature extractors is $91.17 \%$. It is also important to note that the genetically evolved feature extractors used an average of 48.72 patches (See Fig. 6(b)) whereas MLBP used 78 patches (6 patch rows by 13 patch column) (See Fig. 6(a)). The reduction of patches is significant towards the computational time of template matching. Fig. 7 shows the ROC curve of both the MLBP and best evolved feature extractor from GEFE_MLBP on the CASIA dataset. It appears that the MLBP algorithm is outperformed by its GEFE counterpart.

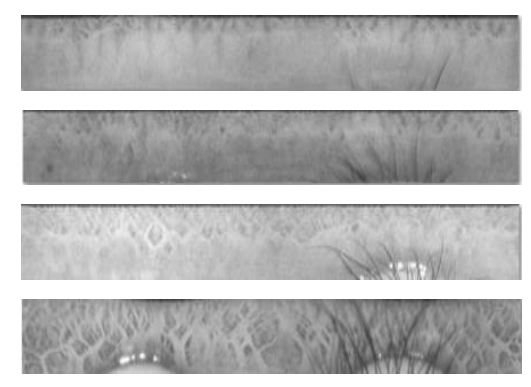

Fig. 5. Some normalized images.

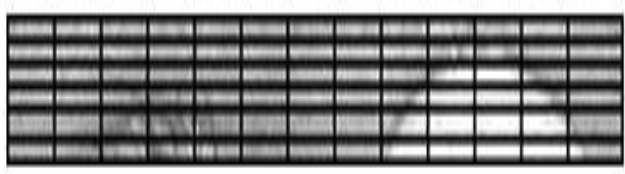

(a)

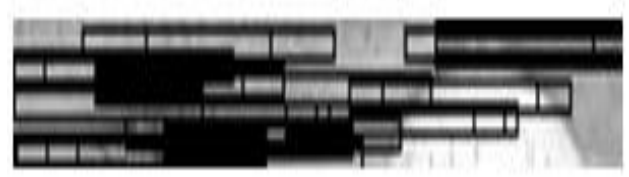

(b)

Fig. 6. (a) Normalized image with 78 patches, (b) normalized image with overlapped patches.

\section{ROC for CASIA Iris Set}

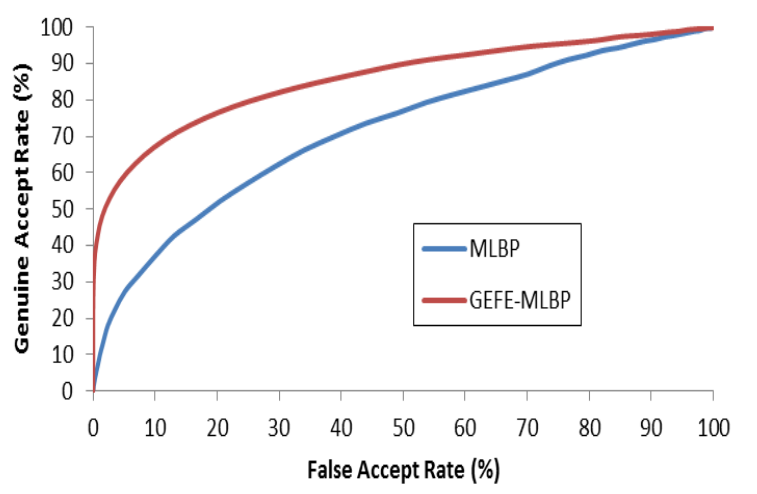

Fig. 7. ROC curve shows the comparison of MLBP and GEFE-MLBP.
TABLE I: RECOGNITION ACCURACIES

\begin{tabular}{lllll}
\hline \hline Method & $\begin{array}{l}\text { Best } \\
\text { Accuracy }\end{array}$ & $\begin{array}{l}\text { Number of } \\
\text { Patches } \\
\text { used }\end{array}$ & $\begin{array}{l}\text { Average } \\
\text { Accuracy }\end{array}$ & $\begin{array}{l}\text { Average } \\
\text { Number of } \\
\text { Patches } \\
\text { used }\end{array}$ \\
\hline MLBP & $86.00 \%$ & 78 & N/A & N/A \\
GEFE_MLBP & $91.17 \%$ & 43 & $88.42 \%$ & 48.72 \\
\hline \hline
\end{tabular}

\section{CONCLUSIONS}

In this research effort, we have achieved two performance goals. First, we propose a new iris segmentation technique based on FCMLS, which is implemented with a new LS formulation and avoids the costly re-initialization process. Thus, it takes less computational time to evolve towards the iris boundary. The proposed FCMLS localizes the iris regions from the degraded eyes accurately. This method appears to have an additional benefit to simplify or eliminate the need for eyelash/eyelid detection as the GEFE and fuzzy LS methods were used. However, further research will be needed to draw a more accurate conclusion. The localization method based on FCMLS avoids the over-segmentation and performs well against the weak iris/sclera boundary since FCMLS combines the merits of traditional fuzzy c-means clustering and LS models. Second, a modified local binary pattern (MLBP), which combines the sign and magnitude features for the improvement of iris texture classification performance, is also applied with the GEFE. Results have shown that by hybridizing MLBP with genetic and evolutionary computations, the recognition accuracy is increased with a reduction in feature usage. Future work will be devoted towards creating more effective fitness functions for GEFE and investigation different feature extraction techniques for iris recognition.

\section{ACKNOWLEDGMENTS}

The authors would like to thank the ARL and the NSF for their support of this research.

\section{REFERENCES}

[1] K. W. Bowyer, K. Hollingsworth, and P. J. Flynn, "Image understanding for iris biometrics: a survey," Comp. Vis. Image Understanding, vol. 110, no. 2, pp. 281-307, 2008.

[2] M. Vatsa, R. Singh, and A. Noore, "Improving iris recognition performance using segmentation, quality enhancement, match score fusion, and indexing," IEEE Trans. Syst. Man and Cyber. B, vol. 38, no. 4, pp. 1021-1035, 2008.

[3] K. Roy, P. Bhattacharya, and C. Y. Suen, "Towards nonideal iris recognition based on level set method, genetic algorithms and adaptive asymmetrical SVMs," Engg. Applications of Artificial Intell., vol. 24, no. 3, pp. 458-475, 2011.

[4] K. Roy, P. Bhattacharya, and C. Y. Suen, "Iris segmentation using variational level set method," Optics and Lasers in Engg., vol. 49, no. 4, pp. 578-588, 2011.

[5] K. Roy and P. Bhattacharya, "Improvement of iris recognition performance using region-based active contour model," Int. J. Pattern Recog. and Artificial Intell., vol. 24, no. 8, pp. 1209-1236, 2010.

[6] J. G. Daugman, "New methods in iris recognition," IEEE Trans. Syst. Man and Cyber. B, vol. 37, no. 5, pp. 1167-1175, 2007.

[7] J. Koh, V. Govindaraju, and V. Chaudhary, "A robust iris localization method using an active contour model and Hough transform," in Proc. IEEE Int. Conf. on Pattern Recog., Istanbul, Turkey, Aug. 2010, pp. 2852-2856. 
[8] S. Shah and A. Ross, "Iris segmentation using geodesic active contours," IEEE Trans. Info. Forensics and Security, vol. 4, no. 4, pp. 824-836, 2009.

[9] B. Li, C. Chui, S. Chang, and S. Hong, "Integrating spatial fuzzy clustering with levelset methods for automated medical image segmentation," Comp. in Biology and Medicine, vol. 11, pp. 1-10, 2011.

[10] T. Ojala, M. Pietikäinen, and T. Mäenpää, "Multiresolution gray-scale and rotation invariant texture classification with Local Binary Pattern," IEEE Trans. on Pattern Analysis and Machine Intelligence, vol. 24, no. 7, pp. 971-987, 2002.

[11] Z. Guo, L. Zhang, and D. Zhang, "A Completed Modeling of Local Binary Pattern Operator for Texture Classification," IEEE Trans. on Image Processing, vol. 19, no. 6, pp. 1657-1663, 2010.

[12] J. Shelton, G. Dozier, K. Bryant, J. Adams, K. Popplewell, T. Abegaz, and K. Ricanek, "Genetic based LBP feature extraction and selection for facial recognition," in Proc. ACM Annual Southeast Regional Conf. 2011, pp. 197-200

[13] J. Shelton, G. Dozier, K. Bryant, L. Smalls, J. Adams, K. Popplewell, and K. Ricanek, "Comparison of genetic-based feature extraction methods for facial recognition," in Proc. Midwest Artificial Intelligence and Cognitive Science Conf., 2011, pp. 216.

[14] J. Shelton A. Alford, T. Abegaz, L. Small, D. Leflore, J. Williams, J.Adams, K. Bryant, G. Dozier, and K. Ricanek, "Genetic \& evolutionary, biometrics: feature extraction from a machine learning perspective," in Proc. IEEE Southeast Conf., 2012.

[15] M. Payne, G. Dozier, J. Shelton, J. Turner, J. Adams, J. Carter, H. Williams, C. Hansen, and I. Dworkin, "Fly wing biometrics using genetic and evolutionary feature extraction," in Proc. IEEE Symposium Series on Computational Intelligence, 2013.

[16] W. Kong and D. Zhang, "Accurate iris segmentation based on novel reflection and eyelash detection model," in Proc. Int. IEEE Sympos. on Intelligent Multimedia, Video and Speech Process., Hong Kong, 2001, pp. 263-266.

[17] K. Chuang, H. Hzeng, S. Chen, J. Wu, and T. Chen, "Fuzzy c-means clustering with spatial information for image segmentation," Computerized Medical Imaging, and Graphics, vol. 30, pp. 9-15, 2006.

[18] C. Li, C. Xu, C. Gui, and M. D. Fox, "Level set evolution without re-initialization: a new variational formulation," in Proc. Comp. Vision and Pattern Recog., 2005.

[19] CASIA-Iris Version 3 interval dataset. [Online]. Available: http://www.cbsr.ia.ac.cn/IrisDatabase.htm

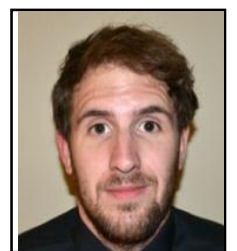

Brian O'Connor is an undergraduate student at North Carolina Agricultural and Technical State University, Greensboro, NC, USA, in the Computer Science Department. Brian is scheduled to graduate with his bachelor's degree in computer science in spring 2014

$\mathrm{He}$ is currently working as a research assistant at North Carolina A\&T and has been in his position for a year. His research interest includes cyber identity, biometrics and machine learning.

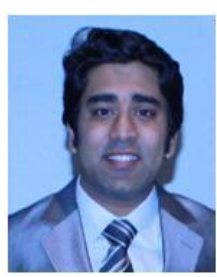

Kaushik Roy received his $\mathrm{PhD}$ from Concordia University, Montreal, QC, Canada in 2011 in Computer Science. He also completed his MS degree in computer science from the Concordia University in 2006 and B.Sc. degree in computer science from University of Rajshahi, Bangladesh in 2001. Kaushik Roy is currently an assistant professor at the Department of Computer Science, and Assistant
Director of the Center for Advanced Studies in Identity Sciences (CASIS), North Carolina A\&T State University, USA. Previously, he worked as a postdoctoral fellow in the Department of Electrical and Computer Engineering, University of Waterloo, ON, Canada during 2011-2012. He also taught at Rajshahi University of Engineering and Technology (RUET) as a lecturer of the Department of Computer Science and Engineering during 2001-2004.

Dr. Kaushik Roy is also the recipient of several fellowships and awards including the prestigious NSERC Visiting Fellowship, FQRNT B3 (Postdoctoral), NSERC (Doctoral) and FQRNT B2 (Doctoral). His research interests include Biometrics, cyber identity, game theory, information fusion, computer vision, machine learning, and pattern recognition. He has published 1 book, 2 book chapters, 11 journal articles and 38 conference articles.

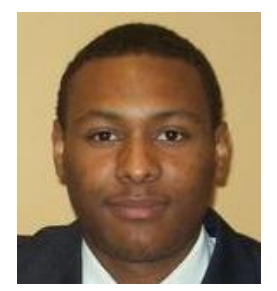

Joseph Shelton is a doctoral student at North Carolina Agricultural and Technical State University, Greensboro, NC, USA, in the Computer Science Department. Joseph obtained both his bachelor's degree and master's degree in computer science at North Carolina A\&T State University in 2010 and 2012

He is currently working as a Research Assistant at North Carolina A\&T and has done so for the last three years. He has helped publish a book chapter in 'New Trends and Developments in Biometrics' titled 'Genetic and Evolutionary Biometrics' and has published over 20 articles in the field of biometrics and genetic \& evolutionary computations. Joseph's research interests include biometrics, cyber security and evolutionary computation.

Mr. Joseph Shelton has received an award for $1^{\text {st }}$ runner up for best student paper at the conference on systems engineering research (CSER 2012)

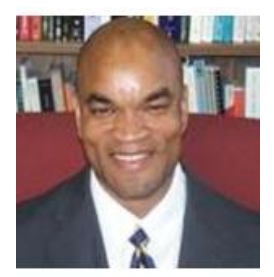

Gerry Vernon Dozier is a professor and the chair of the Computer Science Department at North Carolina A\&T State University. He earned his $\mathrm{Ph} . \mathrm{D}$. from North Carolina State University. He is the director of the Center for Advanced Studies in Identity Sciences (CASIS), as well as the PI for the Center for Cyber Defense (recognized by the National Security Agency and the Department of Homeland Security as a Center for Academic Excellence in Information Assurance Education).

During Gerry's tenure as chair, the department has seen an increase in extramural funding and research publications as well as the establishment of a Ph.D. program. He has also lead in the development of an undergraduate research program where approximately $20 \%$ of the undergraduate students are active participants in funded research projects. Under Gerry's leadership, the NSF Alliance for the Advancement of African American Researchers in Computing (A4RC, www.a4rc.org) experienced a threefold increase (from 6 to 20) in the number of participating universities. A4RC was effective in increasing the number of African-American recipients of advanced degrees in Computer Science.

Dr. Gerry Vernon Dozier has published over 130 conference and journal publications. He has served as an Associate Editor of the IEEE Transactions on Evolutionary Computation and the International Journal of Automation \& Soft Computing. Gerry is also a member of the Editorial Board for the International Journal of Intelligent Computing \& Cybernetics. His research interests include: Artificial \& Computational Intelligence, Genetic Evolutionary, and Neural Computing, Biometrics, Identity Sciences, Cyber Identity, Distributed Constraint Reasoning, Artificial Immune Systems, Machine Learning and Network Intrusion Detection. 